\title{
Detection of recurrent activation patterns across focal seizures: Application to seizure onset zone identification
}

\author{
Manel Vila-Vidal ${ }^{\mathrm{a}, \mathrm{b}, \mathrm{c}}$, Alessandro Principe $^{\mathrm{a}, \mathrm{b}, \mathrm{d}}$, Miguel Ley ${ }^{\mathrm{a}, \mathrm{b}}$, Gustavo Deco ${ }^{\mathrm{c}, \mathrm{e}}$, Adrià \\ Tauste Campo ${ }^{\mathrm{a}, \mathrm{b}, \mathrm{c}, *}$, Rodrigo Rocamora ${ }^{\mathrm{a}, \mathrm{b}, \mathrm{d}, *}$ \\ ${ }^{a}$ Epilepsy Monitoring Unit, Department of Neurology, Hospital del Mar, 08003 Barcelona, Spain \\ ${ }^{b}$ Hospital del Mar Medical Research Institute, 08003 Barcelona, Spain \\ ${ }^{c}$ Center for Brain and Cognition, Department of Information and Communication Technologies, \\ Universitat Pompeu Fabra, 08018 Barcelona, Spain \\ ${ }^{d}$ Faculty of Health and Life Sciences, Universitat Pompeu Fabra, 08003 Barcelona, Spain \\ ${ }^{e}$ Institució Catalana de Recerca i Estudis Avançats, 08010 Barcelona, Spain
}

\begin{abstract}
Objective: We introduce a method that quantifies the consistent involvement of intracranially monitored regions in recurrent focal seizures.

Methods: We evaluated the consistency of two ictal spectral activation patterns (mean power change and power change onset time) in intracranial recordings across focal seizures from seven patients with clinically marked seizure onset zone (SOZ). We examined SOZ discrimination using both patterns in different frequency bands and periods of interest.

Results: Activation patterns were proved to be consistent across more than $80 \%$ of recurrent ictal epochs. In all patients, whole-seizure mean activations were significantly higher for SOZ than non-SOZ regions $(P<0.05)$ while activation onset times were significantly lower for SOZ than for non-SOZ regions $(P<0.001)$ in six patients. Alpha-beta bands $(8-20 \mathrm{~Hz})$ achieved the highest patient-average effect size on the whole-seizure period while gamma band $(20-70 \mathrm{~Hz})$ achieved the highest discrimination values between SOZ and non-SOZ sites near seizure onset $(0-5 \mathrm{~s})$.

Conclusions: Consistent spectral activation patterns in focal epilepsies discriminate the SOZ with high effect sizes upon appropriate selection of frequency bands and activation periods.
\end{abstract}

Significance:The present method may be used to improve epileptogenic identification as well as pinpoint additional regions that are functionally altered during ictal events.

Keywords: Quantitative EEG analysis, Temporal lobe epilepsy, Stereo-EEG

*These authors jointly supervised this work. Corresponding author at: Adrià Tauste Campo, Hospital del Mar Medical Research Institute, Carrer Aiguader, 88, 08003 Barcelona, Spain, adria.tauste@ gmail.com. 


\section{Highlights}

- Consistent recurrent seizures allow to estimate channel activation patterns delineating the seizure onset zone (SOZ).

- The SOZ is characterized by low activation onset times and large time-average activation values.

- SOZ discrimination is maximized below the gamma band when the entire seizure period is considered.

\section{Introduction}

The accurate identification of the epileptogenic zone in patients with medically refractory epilepsy is central to plan an efficient surgical intervention. Yet, after decades of surgical treatment experience, the outcome is not completely successful in a significant proportion of patients (Spencer and Huh, 2008) for several causes including complex epileptogenic networks, surgery technical limitations, among others. Nowadays invasive recording techniques such as stereoencephalography (SEEG) (Munari and Bancaud, 1985; Talairach et al., 1974; Guenot et al., 2002; Engel et al., 2005) provide a continuous monitoring tool for pre-surgical diagnosis that becomes specially effective in patients with challenging focal epilepsies. However, those patients exhibiting complex activation patterns across different seizures still represent a diagnostic challenge because they involve time-consuming EEG evaluations that may lead to inconclusive interpretations. Hence, the use of quantitative tools that assess the consistency of ictal patterns across recurrent seizures might contribute to objectively identify the epileptogenic zone, thus improving pre-surgical diagnosis (Engel et al., 2005) and reducing potential failures. In addition, quantitative measures might provide additional insights into the mechanisms of focal ictogenesis (Goodfellow et al., 2016) when correlated with available structural and functional information.

Over the last decade, several studies have proposed biomarkers to evaluate the epileptogenicity of recorded intracranial structures. A great number of them have analyzed spectral features of SEEG signals (Bartolomei et al., 2008; David et al., 2011; Gnatkovsky et al., 2011, 2014, Andrzejak et al., 2015), which mimic the patterns that are visually identified as epileptogenic during diagnostic EEG inspection (Gnatkovsky et al., 2014; Lagarde et al., 2016). In these works, epileptogenic biomarkers are typically built around two variables, the activation of signal's spectral properties (e.g., the relative amount of signal power in a frequency band (Bartolomei et al., 2008)) and the onset time of this activation, which measure respectively the amount of ictal activity and the degree of early participation of such structures during seizures. Epileptogenicity is then defined for each structure by combining both variables into an index that may be averaged across distinct seizures (Bartolomei et al., 2008; David et al., 2011; Andrzejak et al., 2015) to obtain a single biomarker per region. Critically, these mathematical transformations leading to a single value per region might prove inaccurate when averages are performed across seizures with heterogeneous activation patterns.

In the current study, we developed a flexible, robust and visual-friendly computeraided method to assess the homogeneity of two ictal-driven channel spectral patterns (mean power change and power change onset time) across recurrent focal seizures. We evaluated the method in a group of seven epileptic patients with temporal lobe drugresistant focal seizures. In all patients, we obtained seizure averages of both activation 
patterns for each recorded channel that were represented in two-dimensional plots for complementary clinical evaluation. As an example of application, we used each averaged pattern to characterize the seizure onset zone (SOZ) of seven patients with well identified seizure focus and tested the variability of the results obtained against the main method's parameters. The proposed procedure may be integrated into existing epileptogenic indices by choosing the frequency band of interest and appropriately plugging in each averaged pattern, thus contributing to identify in a robust form central regions in the generation and spread of focal seizures.

\section{Methods}

\subsection{Ethics statement}

All diagnostic and surgical procedures were approved by The Clinical Ethical Committee of our Hospital.

\subsection{Patients and recordings selection}

A total number of 46 focal seizures from seven patients with pharmacoresistant epilepsy were analyzed. A summary of all patients' characteristics is given in Table 1. Seizure onset and termination times of each seizure were independently marked by two epileptologists (RR and AP) using standard clinical assessment. For each seizure we analyzed SEEG recordings from the marked ictal epoch together with $60 \mathrm{~s}$ of pre-ictal and $60 \mathrm{~s}$ of post-ictal epochs.

We selected seven patients in which the seizure focus had been marked by epileptologists under the general principle that "the ictal onset was confined to a certain number of contacts and it was stable through ictal events". Among the selected patients, Patients 1-3 achieved seizure freedom after surgical resection (Engel I) with a follow-up period of at least two years. Patients 4 and 5 underwent radiofrequency thermocoagulation (RFTC, (Bourdillon et al., 2017)) and have showed favorable outcome over the last two years (see Table 1). Patient 4 is now seizure free, while Patient 5 has proven to be responsive to RFTC showing a seizure reduction larger than 50\%. We also selected two patients (Patients 6 and 7) in which the outcome had not been successful because the brain resectomy had failed to completely remove the identified seizure focus. In particular, in Patient 6 the seizure focus could not be removed because it overlapped with eloquent areas.

All recordings were obtained using a standard clinical EEG system (XLTEK, subsidiary of Natus Medical) with a $500 \mathrm{~Hz}$ sampling rate. A uni- or bilateral implantation was performed accordingly, using 5-15 intracerebral electrodes (Dixi Medical, Besançon, France; diameter: $0.8 \mathrm{~mm}$; $5-15$ contacts (or channels), $2 \mathrm{~mm}$ long, $1.5 \mathrm{~mm}$ apart) that were stereotactically inserted using robotic guidance (ROSA, Medtech Surgical, Inc). The decision to implant, the selection of the electrode targets and the implantation duration were entirely made on clinical grounds.

\subsection{Data pre-processing}

EEG signals were processed in the referential recording configuration (i.e., each signal was referred to a common reference). The electrodes per patients included in the analysis are reported in Table 1. All recordings were band-passed filtered (FIR, filter band $[1,165]$ $\mathrm{Hz}$ ) to remove slow drifts and aliasing effects and notch-filtered to remove the effect of the alternate current (Notch FIR filter at $50 \mathrm{~Hz}$ and harmonic frequencies). Channels with artefacts were identified by visual inspection and removed prior to data analysis. For the precise estimation of activation onset times, artefacts simultaneously affecting 
the majority of SEEG channels during short instances were removed using the following systematic procedure. We performed a sliding-window analysis (200 samples width, 1 sample step) along seizure epochs and identified those time windows where the product of the mean correlation and the channel-average signal power was two standard deviations larger than the median.

\subsection{Data analysis}

\subsubsection{Instantaneous activation}

We used the Hilbert transform method (Le Van Quyen et al., 2001; Oweis and Abdulhay, 2011) to obtain a spectrogram of the signal recorded by each channel with a time resolution of $2 \mathrm{~ms}$ (Fig. 1 $\mathrm{A}$ and $\mathrm{B}$ ). The signal was split into non overlapping narrow frequency bands $[f, f+\Delta f]$, with $\Delta f=0.1 f$, starting at $f=3 \mathrm{~Hz}$ and up to $f=160 \mathrm{~Hz}$. Summation of the spectrogram over a given frequency band was performed to collapse the information along one axis, thus obtaining the instantaneous power per channel (Fig. 1 $\mathrm{C}$ ). This step was primarily done over all analyzed frequencies and was later particularized to specific frequency bands. A baseline pre-ictal distribution of power was defined by accumulating the power values of all channels during the first $40 \mathrm{~s}$ of the pre-ictal period. We then normalized each channel's instantaneous power with respect to this baseline and defined the instantaneous activation as the resulting z-score (Fig. $1 \mathrm{E}$ ). The instantaneous activation can be easily interpreted as a measure of the power change during the ictal event. Then, two main measures were defined in this reference space to characterize every channel's ictal activation: the normalized mean activation (nMA) and the activation onset time (AO). Precise definitions of both measures are given in the next sections.

\subsubsection{Normalized mean activation (nMA)}

For every analyzed channel, we defined its mean activation (MA) to be the average of its instantaneous activation during the seizure epoch. This measure quantifies the relative time-average power of every channel during the seizure with respect to the pre-ictal baseline. Although channel mean activations exhibited high variability across seizures, a relative activation profile was commonly preserved (Fig. 2A). Cross-seizure comparisons were then made possible by normalizing the channel's mean activation within each seizure using the z-score, which yielded a normalized mean activation (nMA) value per channel. This normalization step enabled a straightforward comparison of channels' activations from different seizures (Fig. 2B). We then guaranteed the homogeneity of normalized values coming from different seizures. This was achieved by discarding seizures with a fraction equal or greater than 0.15 of channels exhibiting a large deviation $(>2$ standard deviations of the mean) from the median nMA across seizures. Variations of this fraction threshold did not yield significant differences (Fig. 3A). The non-discarded seizures were considered to be part of the normalized seizure ensemble (NSE). Within this ensemble, we then obtained a seizure-average nMA per channel.

\subsubsection{Activation onset time (AO)}

In order to consistently determine channel activation onset times (AO) across seizures, we defined a single activation threshold in the NSE, and translated it to each individual seizure by reverting the initial normalization. The threshold choice was based on the following observation. The activity of channels during seizures exhibited two nMA profiles quite consistently across different patients. One profile was determined by channels of low nMA, which were homogeneously and densely packed in the lowest-value tail of the nMA distribution (Fig. 2D). In contrast, the rest of the channels exhibited high nMA 
values, which were also more variable (Fig. 2D). Based on these distinct groups, we developed an algorithm to identify the low nMA profile channels as the subset of recorded channels with more homogenous activation values ("dense set"). Details of the dense set identification algorithm can be found in the Supplementary Information (section 2.4).

The dense set defined a low nMA distribution. Let $\tilde{\mu}_{D}$ and $\tilde{\sigma}_{D}$ be the mean and standard deviation of this distribution, respectively. Then, the (single) activation threshold in the NSE was set to be $\tilde{d}=\tilde{\mu}_{D}+\tilde{z} \cdot \tilde{\sigma}_{D}$, for some tunable value of $\tilde{z}$ typically ranging from 1 to 3 . By reverting each seizure-specific normalization, we obtained seizure-dependent activation thresholds (Fig. 2D). For every analyzed channel, we defined its activation onset time (AO) as the first time sample where the channel's instantaneous activation crossed the seizure-dependent threshold provided that it remained above this level for at least $95 \%$ of the time during the following $T$ seconds. Results were stable across distinct values of $\tilde{z}(\tilde{z}=1,2,3)$ and $T(T=5 s, 10 s)$. AOs took positive values starting from 0 , which corresponded to the seizure onset time. Channels that never attained the activation threshold were excluded for further AO analysis and representation. Then, AOs from different seizures were pooled together (Fig. 2E and Fig. 2F). For each channel with defined AO in at least half of the seizures, we calculated the seizure average AO (Fig. $2 \mathrm{G}$ ).

\section{Results}

\subsection{Normalized seizure ensemble (NSE)}

In all studied patients, we computed the channels' nMA following the method described above. The homogeneity of normalized values across recurrent seizures was assessed in six patients (Patient 3 had only one seizure) prior to perform seizure averages. In these cases, we identified a NSE composed by a large proportion of focal seizures $(84 \% \pm 6 \%$, mean \pm standard deviation, Table $\mathrm{S} 1)$, which remained stable across a wide range of channel thresholding conditions (Fig. 3A). Furthermore, we could accurately discriminate the high and low nMA profiles in all patients using an in-house set-identification algorithm (see Supplementary Information, section 2.4). Indeed, in all cases this algorithm confined the lowest part of nMA distribution values, in agreement with the initial observation that low nMA channels were more homogeneously distributed than high nMA ones (Fig. 3B).

\subsection{Two-dimensional pattern representation}

In all patients both channel variables (nMA and $\mathrm{AO}$ ) were averaged across seizures within the NSE. To determine the channels' AO, the activation threshold was set at $\tilde{z}=1$ and the time window was set at $T=10 \mathrm{~s}$. Alternative parameter values did not significantly alter the results except in Patient 6 , in which only $\tilde{z}=1$ yielded interpretable AO values due to pre-ictal power changes. Figure 4 represents both averaged patterns in a two-dimensional plot for each patient, which illustrates the different degree of spectral activation that channels exhibited across focal seizures. These plots can be particulized to specific frequency bands or sub-periods of interest within seizures. In particular, one may include variability information (at the expense of readability) by plotting the standard deviation of the estimated patterns across recurrent seizures (Fig. S2). Finally, we tested the extent to which both activation patterns were providing redundant information by computing the Spearman correlation between each variable across all channels and patients (Fig. 4, bottom-right corner). This computation yielded a medium-large correlation value $(-0.64 \pm 0.14$, mean \pm standard deviation $)$, indicating that both variables could provide complementary information. 


\subsection{Application to SOZ identification}

Next, we analyzed how the studied activation patterns correlated with the seizure onset zone in all patients. Specifically, we examined statistical differences in NMAs and AOs between SOZ and non-SOZ channels combining a non-parametric test (Wilcoxon ranksum test) and an effect-size (Cohen's $d$ (Cohen, 1992)) measure (Fig. 5 A, Table S2). In all patients, nMA values were found to be significantly higher for SOZ than non-SOZ regions (Wilcoxon rank-sum test, $P<0.02$ ) and the effect size was found to be very large $(D>2)$ or small-medium $(0.3<D<0.5$, Patient 2$)$. Regarding AO differences, we restricted the comparison to channels with defined onset times (Supplementary Information, Table S3). In all cases except one (Patient 7) all SOZ channels had a defined AO, while in the non-SOZ channels this percentage decreased to $61 \% \pm 20 \%$ (mean \pm standard deviation). In these patients, $\mathrm{AO}$ differences between regions were found to be statistically significant $(P<0.02)$ showing very large effect sizes. Only in Patient 7 the difference was not significant $(P>0.1)$. However, in this patient the percentage of channels with defined AO's was significantly $(P<0.001)$ larger inside the SOZ $(86 \%)$ than outside the SOZ $(16 \%)$.

We demonstrated the flexibility of the method by examining the influence of frequency bands and an initial ictal sub-period into SOZ identification. First, we repeated the whole procedure over pre-selected frequency bands: $\delta-\theta(3-8 \mathrm{~Hz}), \alpha-\beta(8-20 \mathrm{~Hz}), \gamma(20-70 \mathrm{~Hz})$ and supra- $\gamma(70-165 \mathrm{~Hz})$. In all frequency bands, the percentage of seizures included in the NSE was approximately equal to the percentage based on the broadband $(3-165 \mathrm{~Hz})$ spectrum (Supplementary Information, Table S4), although the seizure selection could vary in each band. Then, we compared nMA and AO values for SOZ and non-SOZ channels in the aforementioned bands. Figure $5 \mathrm{~B}$ shows the patient-average effect sizes for these comparisons in each band (see also Supplementary Information, Table S5). The discrimination attained a maximum value in the $\alpha-\beta$ band $(D>2)$ in both variables, with the lowest value being reached in the supra- $\gamma$ band. On the other hand, no discrimination was possible in the supra- $\gamma$ band for AOs, since channels did not have a defined AO for most of the patients. Finally, we analyzed the influence of early ictal activations into SOZ identification by applying the same frequency-dependent analysis over a period spanning $5 \mathrm{~s}$ form seizure onset. Not surprisingly, the patient-average effect size in this case was maximized in the gamma band $(D>3.5)$ and strongly heightened in the supra- $\gamma$ band in agreement with previous studies (Bartolomei et al., 2008; Jirsch et al., 2006).

\section{Discussion}

We proposed a robust methodology to assess recurrent ictal-driven patterns in intracranially recorded signals based on a temporal high-resolution spectral method (Hilbert transform (Le Van Quyen et al., 2001; Oweis and Abdulhay, 2011)). Our approach relies on finding an ensemble of focal seizures for every patient where these patterns can be compared across seizures and then, single biomarkers per channel can be meaningfully obtained to help define the most epileptogenic regions prior to surgery. Based on previous works (Bartolomei et al., 2008; David et al., 2011), we defined ictal-driven activity as an abrupt and sustained change in a the signal power from pre-ictal to ictal epochs. This definition is sufficiently general to make the method adaptive to any frequency band (Gnatkovsky et al., 2011) and ictal sub-period of interest.

\subsection{Relevance of the normalized seizure ensemble and two-dimensional plot}

A primary aspect of our analysis is to prove the existence of a normalized seizure ensemble per patient, which describes the similar activation profile that channels present 
across recurrent focal seizures. As a result, the quantification of channels' ictal activation depends on the activity of the remaining channels during all analyzed seizures. In our study, the normalized seizure ensemble was proven to exist in patients with TLEs (see Table 1). The construction of such ensemble was at expense of leaving out a small percentage of seizures per patient in which a few channels exhibited heterogeneous patterns. Although the treatment of such cases was out of the scope in this study, we speculate that these irregularities might depend on the extent of the area implanted and reflect the variability of the associated paraphysiological activity (e.g. automatisms) (Engel, 2013).

The resulting two-dimensional plot showing activation patterns per channel can serve as a complementary tool in clinical practice to inspect the consistent involvement of all recorded structures in focal seizures. In particular, it may have an impact in the diagnostic procedure by pinpointing regions that require further visual evaluation or that should be included in pre-surgical stimulation sessions.

\subsection{Seizure onset identification and retrospective interpretation}

As an example of application, we used the method to characterize the SOZ of focal seizures in all studied patients. Because of the limiting spatial sampling attained with intracranial EEG, the exact boundaries of the SOZ cannot be in general determined. Yet, the mean activation and the activation onset time can serve as a proxy to localize those channels that are likely to be involved in the earlier stages of the seizure and thus, be more spatially linked to the true underlying SOZ.

Our analysis of nMA and AOs in all patients revealed that the visually marked SOZ involved channels that presented an early increase of the instantaneous power and evolved into largely sustained time-average activation values (Fig. 5A and B). Although both activation patterns provided correlated values (Fig. 4), their complementary use may be diagnostically relevant in special cases such as those of Patients 2 and 7 , in which the level of SOZ discrimination strongly varied across measures.

We considered a cohort of patients that was not homogenous on basis of their postsurgical outcome. In Patients 1 - 5, the proposed operative treatment (either surgery or RFTC) had been compatible with the marked SOZ and thus, the SOZ was assumed to have been correctly mapped. On the contrary, the resected area of Patients $6-7$ had no overlap with the marked SOZ, which posed the question: How reliable is the visually marked SOZ in such cases? Although the relationship between the SOZ resection and the post-surgical outcome is challenged by recent studies (Rummel et al., 2015; Geier et al., 2015; Huang et al., 2012), it is generally agreed that resection of critical parts of the SOZ is a sufficient condition to attain long-term seizure freedom (Rosenow and Lüders, 2001). Under this premise, we first assumed that the true SOZ of these patients had not been resected. Additionally, our analysis showed that the visually marked SOZ in these patients attained similar relative values of nMA and $\mathrm{AO}$ as compared with the initial patients (Fig. 5). All together, these findings strongly suggest that our method could indeed identify the SOZ in all cases and in particular, match the visually marked SOZ that was not resected in the unsuccessful post-surgical cases.

The results on SOZ identification at different frequency bands were shown to conceal with previous works based on gamma-band (Bartolomei et al., 2008; David et al., 2011) and high-frequency oscillations (Malinowska et al., 2015; Jacobs et al., 2016) in short windows after seizure onset. But more importantly, because of the largely sustained activity of SOZ channels suring seizures, our results in the studied patients revealed that low and medium frequency bands characterize SOZ channels when activation measures are averaged along the whole seizure epoch. Further research along this lines may help 
unravel the role of the SOZ during seizure propagation and termination and its interplay with the epileptogenic network.

\subsection{Study limitations}

Our study has some limitations, including the low number of patients with a common epilepsy type (Temporal lobe epilepsy), the spatial sampling inherent to the SEEG technique and a maximum temporal sampling rate of $500 \mathrm{~Hz}$. Further studies should be performed over a larger cohort of patients to determine normalized ensembles across a wider range of seizure typologies. In addition, SOZ and Epileptogenic zone identification based on the studied activation patterns should be assessed in a larger set of patients with long-term seizure freedom.

\section{Conclusions}

The present study proposes a general method to robustly quantify spectral activation patterns that are consistent across recurrent focal seizures. By exploiting the consistent channel activation profiles that patients exhibit across focal seizures, we propose a normalization procedure that allows to jointly analyze channel activation patterns from different seizures. The two-dimensional analysis of the time-average activation and the activation onset time during seizures provides a good characterization of the SOZ that can serve as a complementary and objective measure for surgical planning and retrospective analysis.

\section{Disclosure}

None of the authors has any conflict of interest to disclose. We confirm that we have read the Journal's position on issues involved in ethical publication and affirm that this report is consistent with those guidelines.

\section{Acknowledgments}

The research was supported by the European Union's Horizon 2020 research and innovation program under Grant Agreement No. 720270. M.V. was supported by the Catalan Research project AL08814 - AGAUR - 2014SGR856. G.D. was supported by the European Research Council Advanced Grant DYSTRUCTURE (Grant 295129) and by the Spanish Research Project AEI/FEDER-PSI2016-75688-P. A.T.C. was supported by the European Research Council Advanced Grant DYSTRUCTURE (Grant 295129) and by the Spanish Research Project AEI/FEDER-PSI2016-75688-P.

\section{References}

Andrzejak, R. G., David, O., Gnatkovsky, V., Wendling, F., Bartolomei, F., Francione, S., et al., 2015. Localization of epileptogenic zone on pre-surgical intracranial EEG recordings: toward a validation of quantitative signal analysis approaches. Brain Topogr 28 (6), 832-837.

Bartolomei, F., Chauvel, P., Wendling, F., 2008. Epileptogenicity of brain structures in human temporal lobe epilepsy: a quantified study from intracerebral EEG. Brain 131 (7), 1818-1830.

Bourdillon, P., Isnard, J., Catenoix, H., Montavont, A., Rheims, S., Ryvlin, P., et al., 2017. Stereo electroencephalography-guided radiofrequency thermocoagulation (SEEG-guided RF-TC) in drugresistant focal epilepsy: Results from a 10-year experience. Epilepsia 58 (1), 85-93.

Cohen, J., 1992. A power primer. Psychol bulletin 112 (1), 155.

David, O., Blauwblomme, T., Job, A.-S., Chabardès, S., Hoffmann, D., Minotti, L., et al., 2011. Imaging the seizure onset zone with stereo-electroencephalography. Brain 134 (10), 2898-2911. 
Engel, A. K., Moll, C. K., Fried, I., Ojemann, G. A., 2005. Invasive recordings from the human brain: clinical insights and beyond. Nat Rev Neuro 6 (1), 35-47.

Engel, J., 2013. Seizures and epilepsy. Vol. 83. Oxford University Press.

Geier, C., Bialonski, S., Elger, C. E., Lehnertz, K., 2015. How important is the seizure onset zone for seizure dynamics? Seizure 25, 160-166.

Gnatkovsky, V., Curtis, M., Pastori, C., Cardinale, F., Lo Russo, G., Mai, R., et al., 2014. Biomarkers of epileptogenic zone defined by quantified stereo-EEG analysis. Epilepsia 55 (2), 296-305.

Gnatkovsky, V., Francione, S., Cardinale, F., Mai, R., Tassi, L., Lo Russo, G., et al., 2011. Identification of reproducible ictal patterns based on quantified frequency analysis of intracranial EEG signals. Epilepsia $52(3), 477-488$.

Goodfellow, M., Rummel, C., Abela, E., Richardson, M., Schindler, K., Terry, J., 2016. Estimation of brain network ictogenicity predicts outcome from epilepsy surgery. Sci Rep 6 (29215).

Guenot, M., Isnard, J., Ryvlin, P., Fischer, C., Ostrowsky, K., Mauguiere, F., et al., 2002. Neurophysiological monitoring for epilepsy surgery: The Talairach SEEG method. Stereotact Funct Neurosurg 77 (1-4), 29-32.

Huang, C., Marsh, E. D., Ziskind, D. M., Celix, J. M., Peltzer, B., Brown, M. W., et al., 2012. Leaving tissue associated with infrequent intracranial eeg seizure onsets is compatible with post-operative seizure freedom. J Pediatr Epilepsy 1 (4), 211-219.

Jacobs, J., Vogt, C., LeVan, P., Zelmann, R., Gotman, J., Kobayashi, K., 2016. The identification of distinct high-frequency oscillations during spikes delineates the seizure onset zone better than high-frequency spectral power changes. Clin Neurophysiol 127 (1), 129-142.

Jirsch, J., Urrestarazu, E., LeVan, P., Olivier, A., Dubeau, F., Gotman, J., 2006. High-frequency oscillations during human focal seizures. Brain 129 (6), 1593-1608.

Lagarde, S., Bonini, F., McGonigal, A., Chauvel, P., Gavaret, M., Scavarda, D., et al., 2016. Seizure-onset patterns in focal cortical dysplasia and neurodevelopmental tumors: Relationship with surgical prognosis and neuropathologic subtypes. Epilepsia 57 (9), 1426-1435.

Le Van Quyen, M., Foucher, J., Lachaux, J.-P., Rodriguez, E., Lutz, A., Martinerie, J., et al., 2001. Comparison of Hilbert transform and wavelet methods for the analysis of neuronal synchrony. J Neurosci Met 111 (2), 83-98.

Malinowska, U., Bergey, G. K., Harezlak, J., Jouny, C. C., 2015. Identification of seizure onset zone and preictal state based on characteristics of high frequency oscillations. Clin Neurophysiol 126 (8), 15051513.

Munari, C., Bancaud, J., 1985. The role of stereo-EEG in the evaluation of partial epileptic seizures. The epilepsies. London: Butterworths, 267-306.

Oweis, R., Abdulhay, E., 2011. Seizure classification in EEG signals utilizing Hilbert-Huang transform. Biomed Eng online 10 (1), 1.

Rosenow, F., Lüders, H., 2001. Presurgical evaluation of epilepsy. Brain 124 (9), 1683-1700.

Rummel, C., Abela, E., Andrzejak, R. G., Hauf, M., Pollo, C., Müller, M., et al., 2015. Resected brain tissue, seizure onset zone and quantitative EEG measures: towards prediction of post-surgical seizure control. PloS one 10 (10), e0141023.

Spencer, S., Huh, L., 2008. Outcomes of epilepsy surgery in adults and children. Lancet Neurol 7 (6), 525-537.

Talairach, J., Bancaud, J., Szikla, G., Bonis, A., Geier, S., Vedrenne, C., 1974. New approach to the neurosurgery of epilepsy. stereotaxic methodology and therapeutic results. 1. introduction and history. Neurochirurgie 20,1 . 







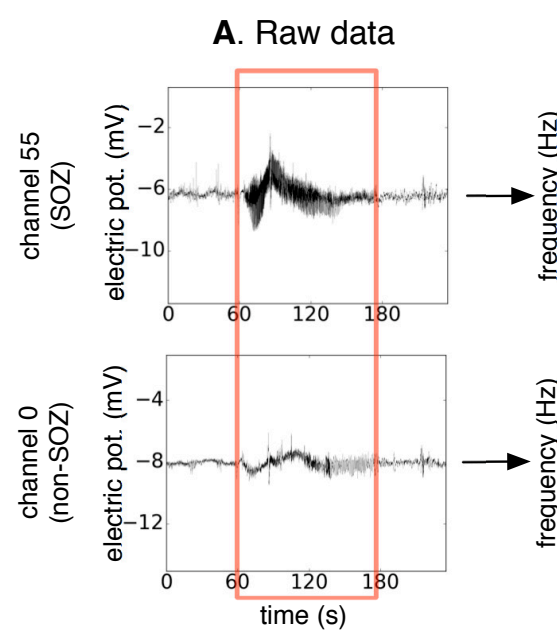

D. Raw data

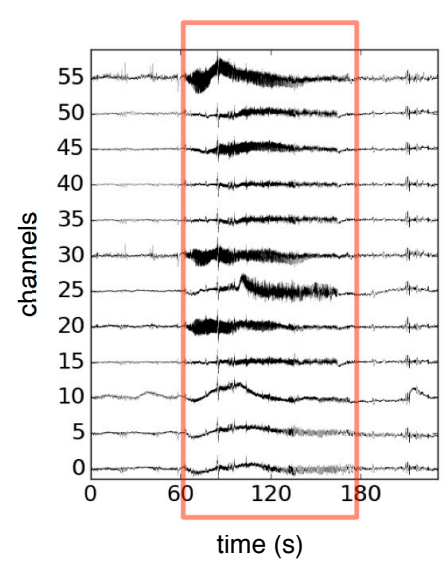

B. Spectrogram


E. Instantaneous activation

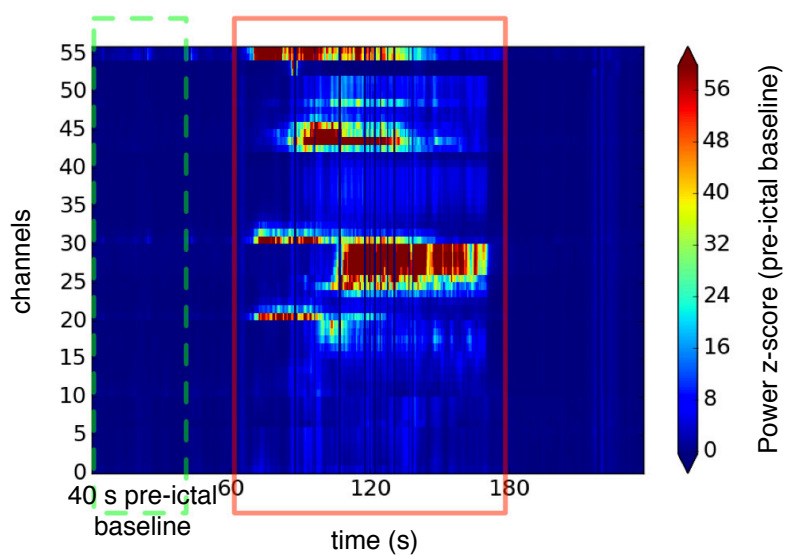

Figure 1: Schematic illustration showing the computation of channels' instantaneous activation in one seizure (first recorded seizure of patient 1). (A) SEEG recordings from two channels labelled as 55 (SOZ) and 0 (non-SOZ). The seizure epoch is indicated with a red frame, leaving 60 seconds of pre-ictal and post-ictal periods for comparison. (B) Spectrograms obtained with the Hilbert transform method (keeping the time resolution equal to the sampling rate). (C) Frequency-independent instantaneous power obtained by summation of the instantaneous power over all frequencies. (D) SEEG recordings from a selection of channels. (E) Time-varying instantaneous activation for 56 channels, obtained by normalization of the frequency-independent instantaneous power with respect to a baseline distribution defined by the power values of all channels during the first 40 seconds of the pre-ictal period. The five implanted electrodes correspond to the following sequences of channels: Fc (0-9), A (10-19), Ha (54,55,20-29), Hp (30-41) and TP (42-53). Lowest numbers within an electrode correspond to the deepest intracerebral channels. 
A. MA (several seizures)



B. nMA (all seizures)

(in the NSE)

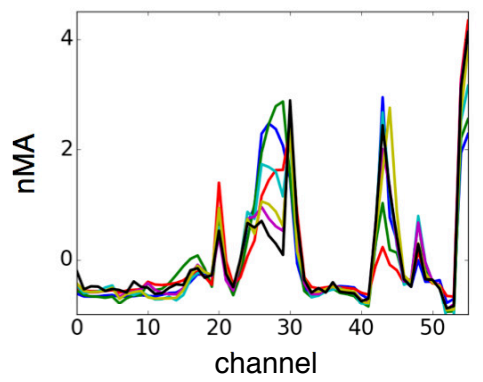

C. Seizure-average nMA

(in the NSE)

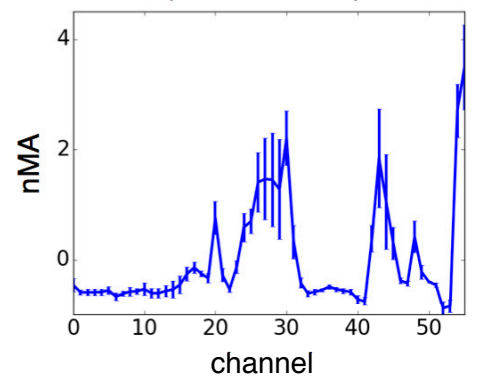

\section{Setting an activation threshold}

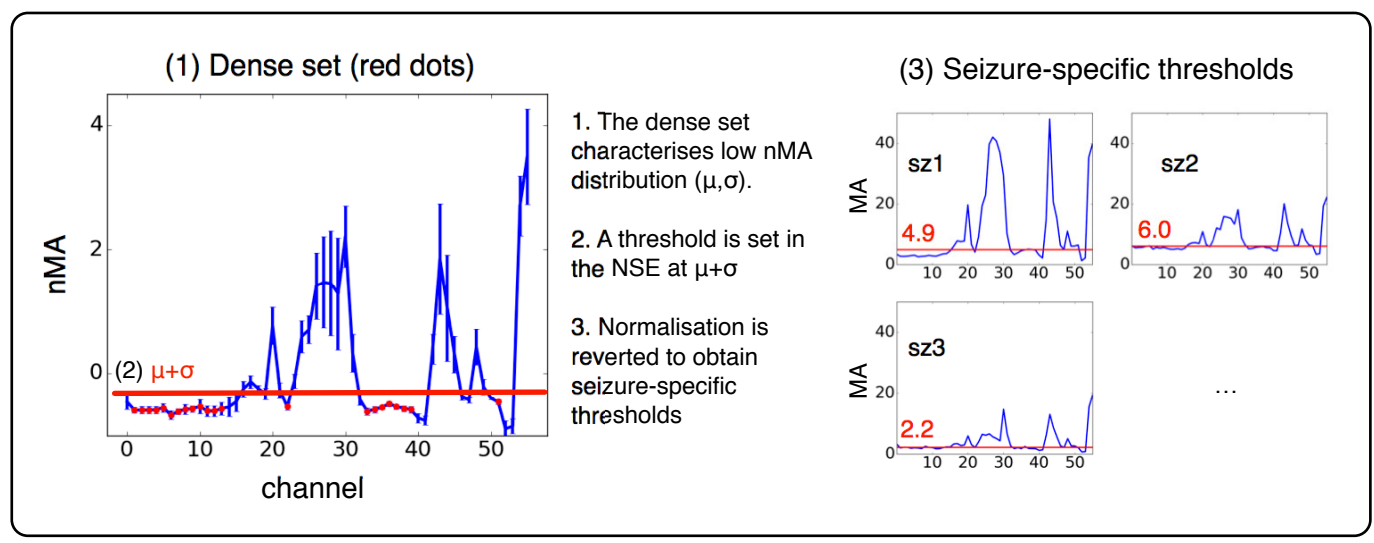

\section{E. AO (several seizures)}

F. AO (all seizures)

G. Seizure-average AO
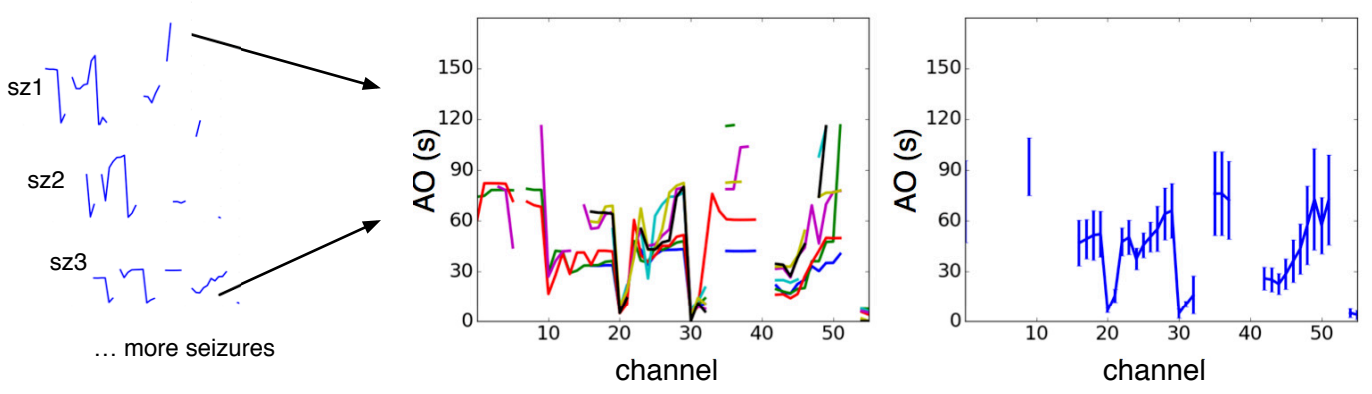

Figure 2: (A) Mean activation (MA) profiles for some exemplary seizures. MA was defined as the average instantaneous activation during the seizure period. (B) Normalized mean activation (nMA), obtained by normalization of channels' MA within each seizure using the z-score. (C) Seizure-average nMA in the NSE. Error bars show one standard deviation. (D) In order to consistently determine channel activation onset times (AO) across seizures, we defined a single activation threshold in the NSE using an own developed algorithm (See S.I.) and translated it to each individual seizure by reverting the initial normalisation. (E) AO profiles for some exemplary seizures. For each analyzed channel, we defined its AO as the first time sample where the channel's instantaneous activation crossed the threshold provided that it remained above this level for at least $95 \%$ of the time during the following $T$ seconds ( $T=10 \mathrm{~s}$ in this case). AO took positive values starting from 0 , which corresponded to the seizure onset time. Channels that never attained the activation threshold were not assigned a defined AO. (F) AO for homogeneous seizures. (G) Seizure average $\mathrm{AO}$ over channels with a defined $\mathrm{AO}$ in at least half of the seizures. 
A. NSE seizures (patient average)

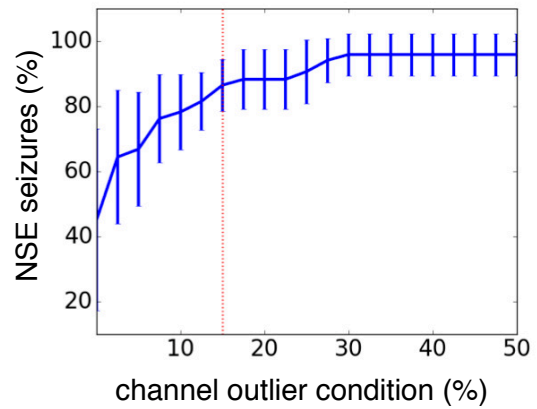

B. Dense set nMA

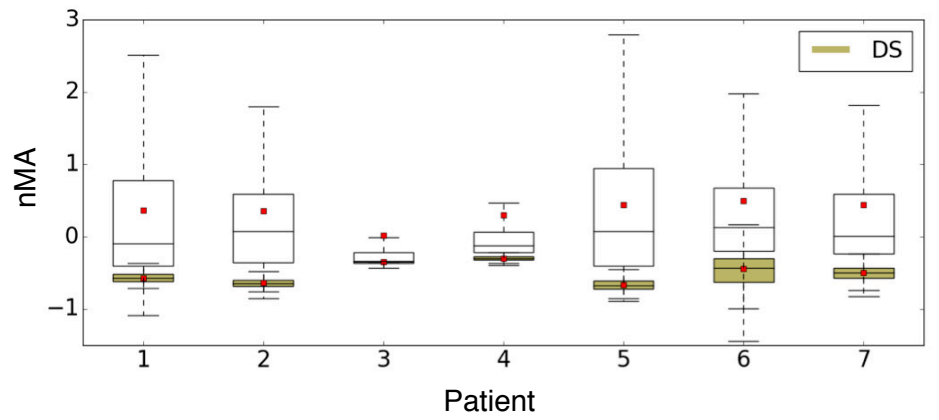

Figure 3: Homogeneity of the normalised seizure ensemble (NSE). (A) For each thresholding value $c$, we show the average percentage (across patients) of seizures with a fraction of highly deviating channels $(>2$ standard deviations of the mean) smaller than $c$. Error bars denote the standard deviation. In our analysis, we chose the threshold value 0.15 because the curve started to stabilize at this value. For this choice, we identified a non-negligible subset of focal seizures for every patient $(84 \% \pm 6 \%$, mean \pm standard deviation) with minimal variability of nMA across seizures. (B) nMA distribution within the dense set and its complementary. In all cases, the algorithm confined the lowest part of nMA distribution values, in agreement with the initial observation that low nMA channels were more homogeneously distributed than high nMA ones. 

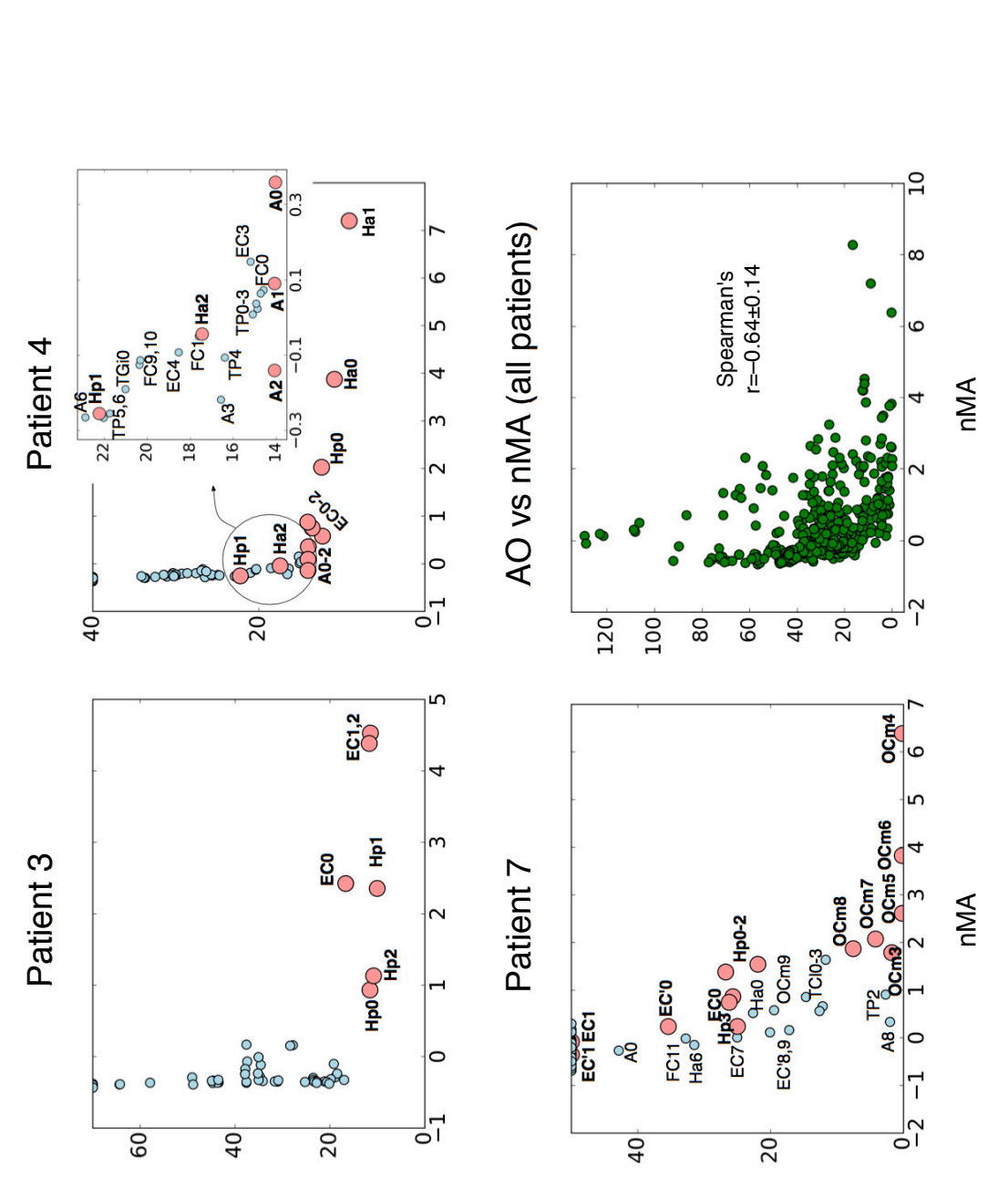

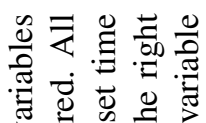

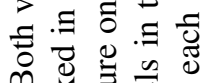

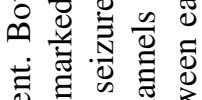
氧.

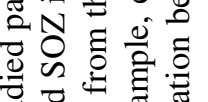

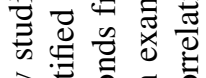

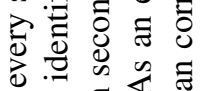

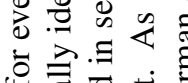

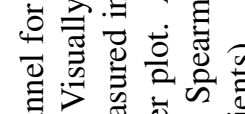

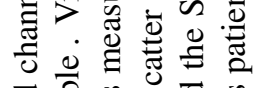
可

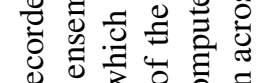

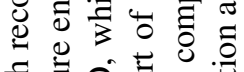

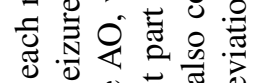
क्ष

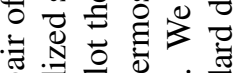
善券

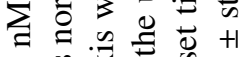

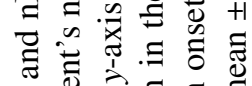



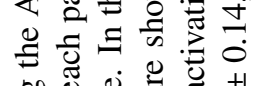
on
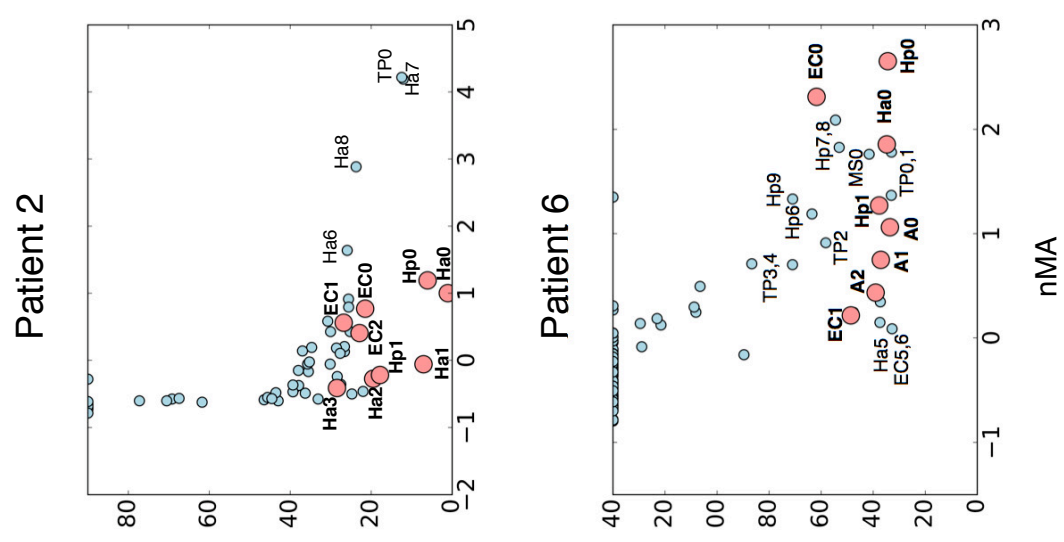

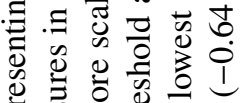

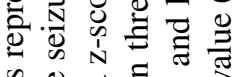

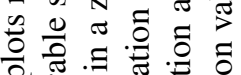

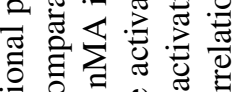

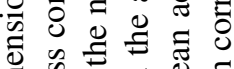

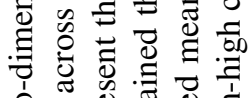

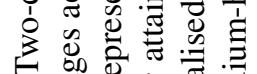
.

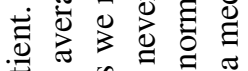

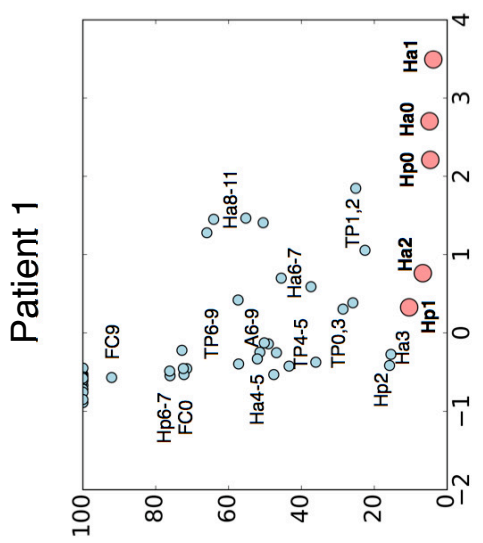

(s) $\mathrm{OH}$

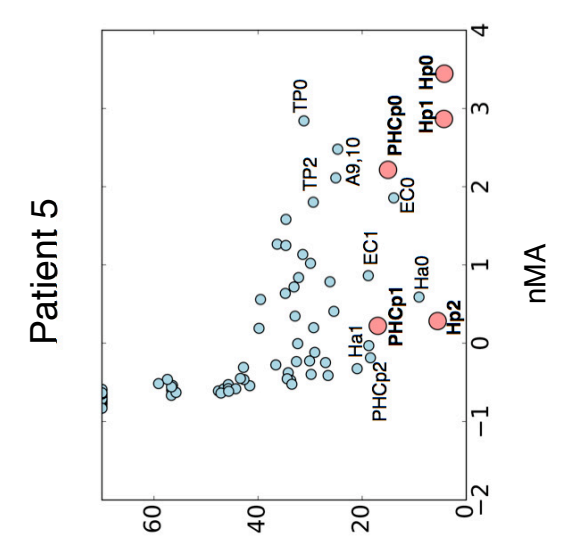

(s) $\mathrm{OH}$
믈.

造

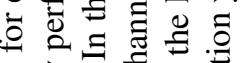
叁家踏 $\Xi$

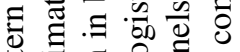

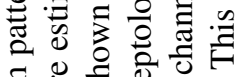

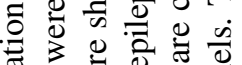

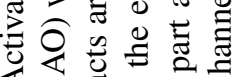

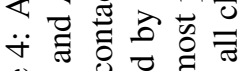

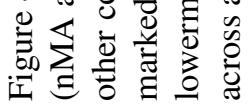


A. SOZ vs non-SOZ, seizure-average nMA and $A O$
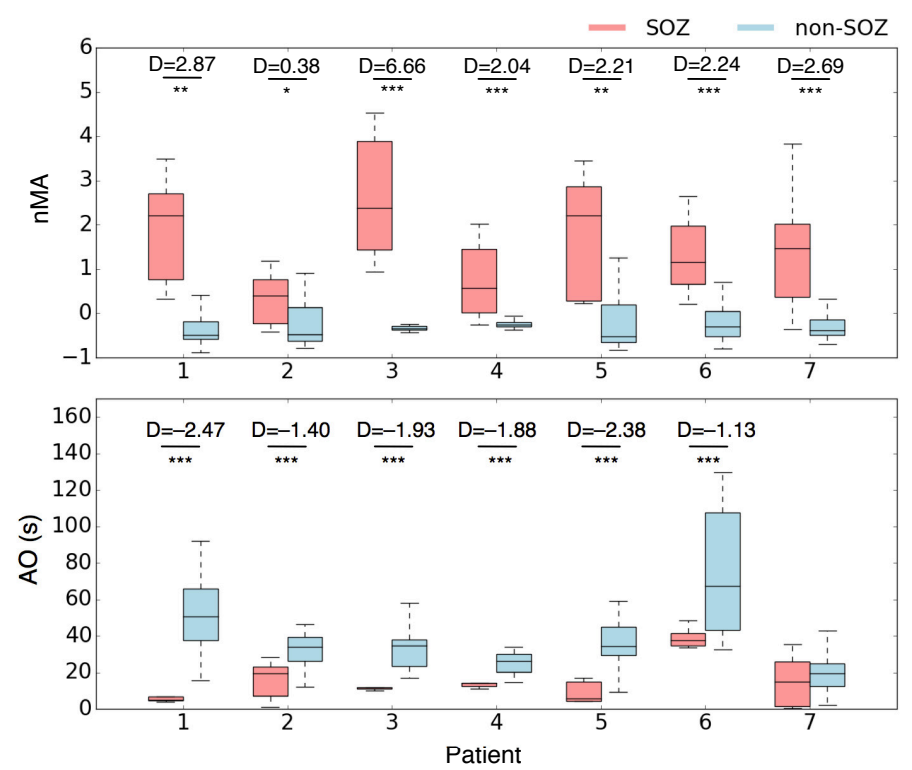

B. Effect sizes for different parameters (patient-average)

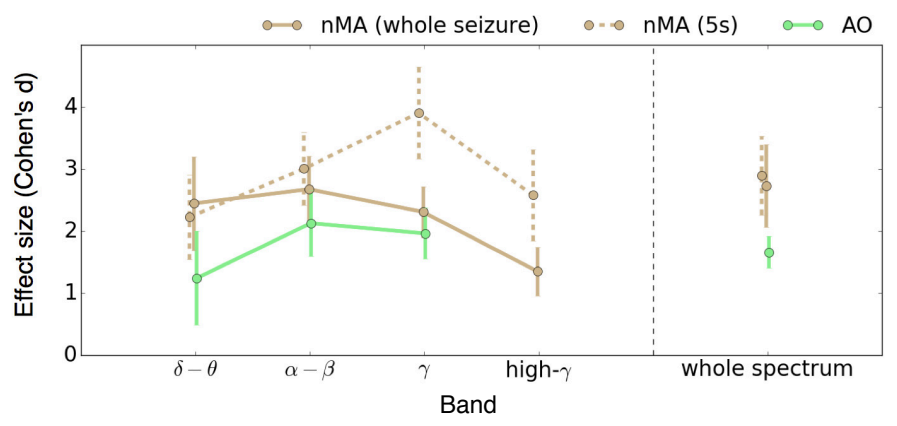

Figure 5: Activation patterns identify the SOZ. (A, top) Boxplots showing the distribution of nMA values across SOZ and non-SOZ groups. All group differences were statistically significant (Wilcoxon rank-sum test, $P<0.02)$ and its effect size (Cohen's $d$, reported in the figure) was very large $(D>2)$ in all cases except in Patient $2(D=0.38)$. Group (SOZ/non-SOZ) sample sizes for each patient were 5/51, 9/58, $6 / 53,11 / 67,5 / 80,8 / 96$ and 14/93. (A, bottom) Boxplots showing the distribution of defined AO values values across SOZ and non-SOZ groups. In Patients 1-6, AO differences between both sets were statistically significant $(P<0.02)$ and its effect size (Cohen's $d$, reported in the figure) was very large $(D>1)$. In Patient 7, the difference was not significant $(P>0.1)$, but the percentage of channels with defined AO's inside the SOZ (86\%, Table S2) was significantly larger (Wilcoxon ranksum test, $P<0.05, N_{1}=14, N_{2}=93$ ) than outside the SOZ (16\%, Table S2). Group (SOZ/non-SOZ) sample sizes for each patient were 5/29, 9/42, $6 / 45,11 / 41,5 / 55,8 / 22,12 / 15$. (B) Patient-average SOZ discrimination values as a function of distinct frequency bands (whole seizure and 5 seconds initial sub-period). For every patient, nMA and AO values for SOZ and non-SOZ channels were obtained upon filtering EEG signals in the bands $\delta-\theta(3-8 \mathrm{~Hz})$, $\alpha-\beta(8-20 \mathrm{~Hz}), \gamma(20-70 \mathrm{~Hz})$ and supra- $\gamma(70-165 \mathrm{~Hz})$, respectively, and following the main procedure. Each curve shows the patient average effect sizes per band. No comparison of AO values was possible in the supra- $\gamma$ band since channels did not have a defined AO for most of the patients. 